\title{
Characterization of an endophytic Gloeosporium sp. and its novel bioactivity with "synergistans"
}

\author{
Authors: George A. Schaible, Gary A. Strobel, \\ Morgan Tess Mends, Brad Geary, and Joe Sears
}

The final publication is available at Springer via http://dx.doi.org/10.1007/s00248-014-0542-y.

Schaible, George A., Gary A. Strobel, Morgan Tess Mends, Brad Geary, and Joe Sears. Characterization of an Endophytic Gloeosporium sp. and Its Novel Bioactivity with "Synergistans". Microbial Ecology. December 2014. Pages 1-10. https://dx.doi.org/10.1007/s00248-014-0542-y

Made available through Montana State University's ScholarWorks scholarworks.montana.edu 


\title{
Characterization of an endophytic Gloeosporium sp. and its novel bioactivity with "synergistans"
}

\author{
George A. Schaible ${ }^{1}$, Gary A. Strobel ${ }^{1 *}$, Morgan Tess Mends ${ }^{1}$, Brad Geary ${ }^{2}$ and \\ Joe Sears ${ }^{3}$ \\ ${ }^{1}$ Department of Plant Sciences, Montana State University, Bozeman, Montana 59717, USA \\ ${ }^{2}$ Department of Botany and Wildlife Sciences, Brigham Young University, Provo, Utah, 84602 \\ ${ }^{3}$ Center for Lab Services/RJ Lee Group, 2710 North 20 ${ }^{\text {th }}$ Ave., Pasco, Washington 99301, USA
}

Key words: Synergistans, 6-Pentyl-2H-pyran-2-one, Gloeosporium sp., Endophytes, $\mathrm{mSE}_{50}=20$ median Synergistic Effect

*Corresponding Author: Gary Strobel; email uplgs@montana.edu 


\section{Abstract}

Gloeosporium sp. (OR-10) was isolated as an endophyte of Tsuga heterophylla (Western hemlock). Both ITS and $18 \mathrm{~S}$ sequence analyses indicated that the organism best fit either Hypocrea spp. or Trichoderma spp., but neither of these organisms possess conidiophores associated with acervuli, in which case the endophytic isolate OR-10 does. Therefore, the preferred taxonomic assignment was primarily based on the morphological features of the organism as one belonging to the genus Gloeosporium sp. These taxonomic observations clearly point out that limited ITS and $18 S$ sequence information can be misleading when solely used in making taxonomic assignments. The volatile phase of this endophyte was active against a number of plant pathogenic fungi including Phytophthora palmovora, Rhizoctonia solani, Ceratocystis ulmi, Botrytis cinerea and Verticillum dahliae. Among several terpenes and furans, the most abundantly produced compound in the volatile phase was 6-pentyl-2H-pyran-2-one, a compound possessing antimicrobial activities. When used in conjunction with $\mu \mathrm{l}$ amounts of any in a series of esters or isobutyric acid, an enhanced inhibitory response occurred with each test fungus that was greater than that exhibited by Gloeosporium sp. or the compounds tested individually. Compounds behaving in this manner are hereby designated "synergistans." An expression of the "median Synergistic Effect," under prescribed conditions, has been termed the $\mathrm{mSE}_{50}$. This value describes the amount of a potential synergistan that is required to yield an additional median $50 \%$ inhibition of a target organism. In this report, the $\mathrm{mSE}_{50} \mathrm{~S}$ are reported for a series of esters and isobutyric acid. The results indicated that isoamyl acetate, allyl acetate and isobutyric acid generally possessed the lowest $\mathrm{mSE}_{50}$ values. The value and 
potential importance of these microbial synergistic effects to the microbial environment are also discussed.

\section{Introduction}

Endophytes are microorganisms that reside symbiotically to borderline pathogenically in virtually every plant without causing any externally apparent signs or symptoms of their presence [14, 23, 27]. In exchange for the host providing an appropriate environment for the endophyte, the fungal endophyte will produce volatile organic compounds (VOCs) that ward off pathogenic microorganisms $[14,16,23,27]$. Because of the production of VOCs, endophytes allow their host plants to withstand attacks by pathogens as well as harsh environments, including drought and salinity [23]. These attributes of endophytic fungi make them of great interest for further research and analysis $[1,4,5,14,15,16,21]$. As an example, the VOCs produced by the endophytes, once purified and characterized are being examined for uses in medicine, agriculture, and industry. Because of the diversity of these unique attributes and their ability to produce bioactive molecules, endophytes are critically important and highly sought after.

The isolate, designated OR-10 and characterized as Gloeosporium sp., is a fungal endophyte isolated from small stems of Tsuga heterophylla (Western hemlock) sampled in the Pacific Northwest. The organism was initially shown to produce 6-pentyl-2H-pyran-2-one, which possesses some microbial inhibitory properties and is a product of certain Trichoderma spp. [8, 11]. In this study, tests were performed to learn if the VOCs made by Gloeosporium sp. behave in a synergistic manner when supplemented with known compounds, such as small molecular 
weight acids and esters that had been previously shown to exhibit such activities [19]. For instance, in the case of Oidium sp., which makes a family of related esters possessing some volatile inhibitory activities, the addition of isobutyric acid as a supplement to the fungal culture enhanced its fungal inhibitory properties, causing a more extensive inhibition of the test organism than either the fungal or the acid could produce alone [19]. Such activity is deemed synergism and has been observed as a tactical method to inhibit pathogen growth $[12,19,22$,

24]. Not all compounds have this inherent property, especially as it relates to microbial inhibitory properties. For this purpose, the term "synergistan" is now proposed as a property possessed by any chemical compound when placed with another, resulting in microbial inhibitory activity greater than either compound alone. This report shows that the endophytic Gloeosporium sp. possesses VOCs with antimicrobial activity. However, in addition, certain small molecular weight compounds (synergistans), when placed with the fungus culture, yielded an added degree of antimicrobial activity to the combined gas phase of the fungus and the synergistans. A novel expression of this synergistic activity has been termed the median Synergistic Effect $\left(\mathrm{mSE}_{50}\right)$, and it, too, is described in this report. Finally, the importance of this phenomenon to microbial ecology is also discussed.

\section{Materials and Methods}

\section{Isolation, culturing and storing of Or-10}

The fungal endophyte used in this study was collected in coastal Washington State at $46^{\circ} 38^{\prime} \mathrm{N}$, $123^{\circ} 55^{\prime} \mathrm{W}$ from the branches of Tsuga heterophylla. Branch samples were dipped in $100 \%$ 
ethanol and flamed for surface decontamination. To culture the endophytes living in the plant material, tissue was cut from the epidermal and pith tissues of the plant with a sterilized surgical scalpel, and the pieces were incubated at room temperature $\left(23^{\circ} \mathrm{C}\right)$ on water agar (WA) for ten days with alternating 12 hour periods of light and dark exposure $[1,4,9]$. The endophyte designated OR-10 was hyphal tipped from the medium after growth from the plant material and was incubated on potato dextrose agar (PDA). After ten days, strong organic odors were being emitted by the culture. A culture of the endophyte was preserved by incubating it at room temperature on sterilized barley seeds that were then placed in a cryogenic vial containing $10 \%$ glycerol and frozen at $-80^{\circ} \mathrm{C}$ in Montana State University's living mycological culture collection as acquisition No. 2401.

\section{Morphological and phylogenetic identification of OR-10}

The endophytic fungus OR-10 was grown on WA with a small amount of sterilized branch material from its host plant (Tsuga heterophylla) for fifteen days to allow the formation of fruiting structures. Fruiting structures eventually arose on the plant material, and it was placed in filter paper packets and suspended in $2 \%$ glutaraldehyde in $0.1 \mathrm{M}$ sodium cacodylate buffer ( $\mathrm{pH}$ 7.2-7.4) with Triton $\mathrm{X}$ (wetting agent), as previously described [7]. For scanning electron microscopy (SEM), the fungal material was dried to the critical point, gold sputter coated, and images were recorded with an XL30 ESEM FEG in high vacuum mode using the EverhartThornely detector [7].

Phylogenetic analysis of OR-10 was carried out by the attainment of the internal transcribed spacer (ITS) and $18 \mathrm{~S}$ ribosomal gene sequences. The fungus was grown on PDA for 7 days, after 
which the genomic DNA was extracted using the Prepman Ultra Sample Preparation Reagent (Applied Biosystems, USA), according to the manufacturer's guidelines. The ITS regions of the fungus were amplified with the universal ITS primers, ITS1 (5'-TCCGTAGGTGAACCTGCGG-3') and ITS4 (5'-TCCTCCGCTTATTGATATGC-3') using polymerase chain reaction (PCR) $[25,26]$. The $18 \mathrm{~S}$ regions were amplified using the universal $18 \mathrm{~S}$ primers, NS3 (5'GCAAGTCTGGTGCCAGCAGCC-3') and NS4 (5'-CTTCCGTCAATTCCTTTAAG-3') [25, 26]. The PCR conditions used were as follows: initial denaturation at $94^{\circ} \mathrm{C}$ for $3 \mathrm{~min}$ followed by 30 cycles of $94^{\circ} \mathrm{C}$ for $15 \mathrm{~s}, 50^{\circ} \mathrm{C}$ for $30 \mathrm{~s}, 72^{\circ} \mathrm{C}$ for $45 \mathrm{~s}$, and a final extension at $72^{\circ} \mathrm{C}$ for $5 \mathrm{~min}$. The amplified product $(5 \mu \mathrm{l})$ was visualized on $1 \%(\mathrm{w} / \mathrm{v})$ agarose gel to confirm the presence of a single amplified band. The amplified products were purified using the Qiagen MinElute PCR Purification Kit (Qiagen Company, Germany). The purified products were sequenced by UCBerkeley DNA Sequencing Facility and the sequences were aligned to other GenBank sequences using the BLASTN program to ascertain the sequence homology with closely related organisms [2].

\section{Identification of VOCs}

Solid-phase micro-extraction gas chromatography and mass spectroscopy (SPME-GC/MS) was performed on the head space above a ten-day old culture of OR-10 grown on PDA in a Petri plate containing PDA as previously described [4]. The gas chromatograph was interfaced to a Hewlett-Packard 5973 mass selective detector (mass spectrometer) operating at unit resolution. Data acquisition and data processing were performed on the Hewlett-Packard CHEMSTATION software system. Initial identification of the unknown organic gases produced 
by OR-10 was made through library comparison using the National Institute of Standards and Technology (NIST) database [4, 7]. Comparative analyses were conducted on control Petri plates containing only PDA. The compounds obtained from the control plate (PDA alone) were mostly styrene and a number of benzene derivatives. These compounds were removed from the data set obtained from the GC/MS of the fungal culture. Any compounds that were under a quality match of $70 \%$ were deemed unknown.

\section{Gas phase bioassay tests for volatile antimicrobials}

Bioassay tests were performed to assess the inhibitory activity of OR-10 against microbes using a gas phase bioassay test as previously described [17]. The test microbes that were used in this assay are as follows; Phytophthora palmivora, Rhizoctonia solani, Ceratocystis ulmi, Colletotrichum lagenarium, Verticillium dahlia, Botrytis cinerea, Sclerotinia sclerotiorum, Pythium ultimum, Trichoderma viridae, Cercospora beticola, Geotrichium candidum, Aspergillus fumigatus, and Fusarium solani (Table 2). To perform the gas phase bioassay, a $2.5 \mathrm{~cm}$ wide strip of agar was removed from the middle of the Petri plate, leaving two half-moon sections of PDA [17]. This allowed the VOCs produced by OR-10 to interact with the microbes exclusively by gas phase with no contact through the agar medium. Next, OR-10 was inoculated on one of the crescent pieces of PDA for 10 days at $23^{\circ} \mathrm{C}$ to allow the organism to mature for the optimal production of VOCs. A plug of PDA $(5 \times 5 \times 5 \mathrm{~mm})$ containing the test microbe was placed on the half-moon section on the opposite side of the plate, then the petri plate was sealed and incubated for 48 hours at $23^{\circ} \mathrm{C}$. After incubation, the radial growth of the mycelium from the test fungus was measured and recorded for comparison to the control, which was a PDA plate 
containing only the untreated test fungus. The test with each designated assay organism was repeated three times for statistical relevance.

\section{Antimicrobial synergism of OR-10 with esters and acids}

Antimicrobial synergism between OR-10 and other compounds was tested under the conditions described above. Compounds suspected of being synergistans were esters as well as isobutryic acid selected, in part, on the basis of their presence in other VOC-producing bioactive fungi [7, $9,17]$. These compounds included allyl acetate, n-decyl acetate, hexyl acetate, isobutyl acetate, isoamyl acetate, and phenethyl acetate. They were tested for their potential ability to inhibit the fungal pathogens Botrytis cinerea, Rhizoctonia solani and Ceratocystis ulmi which were selected because of their sensitivity to OR-10. The tests were run by incubating OR-10 on one half of a PDA plate for ten days, after which the test fungus was inoculated on the other half of the plate. The ten-day incubation period allowed OR-10 to reach optimal VOC production. A microwell was placed in the gap between the two half-moon pieces of PDA and an aliquot (ranging from $0.5 \mu \mathrm{L}$ to $10 \mu \mathrm{L}$ ) of the appropriate ester or acid was added. The microwell kept the test compound from diffusing directly into the medium, while still allowing it to enter the gas phase and interact with the VOCs produced by OR-10. The tests were run for 48 hours at $23^{\circ} \mathrm{C}$, after which the radial growth of the mycelia from the fungal test pathogens was measured. The assays were compared to three different PDA plate controls, including one containing only the test fungus (i.e. fungi pathogens), one with a microwell containing the test compound and the test fungus, and one with a gas phase assay, where OR-10 was inoculated for ten days on one PDA crescent and the test fungus was inoculated on the opposite crescent 
with no test compound present. Assays were repeated at least three times for each condition. A two-tail T-test was used to determine the statistical relevant difference between the effect of the synergistan alone against the pathogen and the synergistan in the presence of OR-10 against the pathogen and duly reported in the results section.

\section{Results}

\section{Classification of endophytic isolate OR-10 as a member of Gloeosporium}

Morphological analysis of the isolated fungal endophyte OR-10 shows that this species fits the description of the fungal genus - Gloeosporium. When grown on PDA, the fungus exhibited white, creamy, non-aerial hyphae, and acervuli fruiting bodies formed when the fungus was grown on WA in the presence of its host plant material. The cultural characteristics were notably distinct and different from Trichoderma sp., which commonly displays a proliferation of rapidly growing aerial hyphae possessing a greenish tinge when grown at $23^{\circ} \mathrm{C}$ with day/night periods. When inoculated on PDA, Trichoderma sp. grows rapidly and covers the surface of the PDA on a 100mm petri dish within 2 days. After ten days on WA, Trichoderma sp. begins to form fruiting structures, something OR-10 commonly does only in the presence of its host plant material. Scanning electron micrographs of OR-10 grown on host plant material revealed acervuli with a size range of $450 \times 500 \mu \mathrm{m}$ and spore sizes of $3 \times 4 \mu \mathrm{m}$ (Fig. 1). Unfortunately, many of the spores collapsed due to the fixation process, but some spores maintained their cell wall integrity (Fig. 1D). The fruiting structure morphology of OR-10 seen in the micrographs fits the description found in the literature of the characteristics of Gloeosporium sp., including 
fruiting structures that form on host material $[3,13]$. The morphological appearances of OR-10 sharply contrast those commonly observed in Trichoderma spp.

\section{Phylogenetic identity of OR-10 by analysis of ITS-5.8S rDNA and $18 \mathrm{~S}$ rDNA}

The ITS-5.8S rDNA sequence of OR-10 was amplified using the ITS1 and ITS4 primers, yielding a sequence of 551 base pairs. BLAST analysis of the ITS rDNA revealed that OR-10 was most phylogenetically similar to Trichoderma sp. (accession number KF367557.1) with an E value of zero. In addition to the ITS analysis, the $18 \mathrm{~S}$ rDNA region was examined for a greater coverage of the genome. The 18S rDNA region was amplified using NS3 and NS4 primers and yielded a sequence of 531 base pairs. The BLAST analysis of this region resulted in the sequence being most phylogenetically similar to a Hypocrea nigricans strain FD12 18S ribosomal RNA gene (accession number KF527499.1), also with an E value of zero. Both 18S rDNA and ITS-5.8S rDNA of Gloeosporium sp. OR-10 have been submitted to GenBank with the assigned accession numbers ITS=KM261839 and 18S=KM261840. Both Trichoderma sp. and Hypocrea sp. are known telemorphs of each other that make freely-formed ascospores and conidiophores, respectively. Although ITS and $18 \mathrm{~S}$ regions of the genome are used as phylogenetic marker genes, they provide little resolution to separate species, in contrast to whole genome comparisons.

Because OR-10 produces acervuli and not ascospores or conidiospores on freely formed conidiophores, it can be concluded that the isolated endophyte OR-10 is neither a Hypocrea sp. nor Trichoderma sp., although the ITS and 18S sequence data suggest otherwise. Although the fungal endophytic isolate OR-10 may be phylogenetically similar to Trichoderma and Hypocrea 
based on the ITS-5.8S and $18 \mathrm{~S}$ regions, it is not morphologically or phenotypically similar in any cultural or structural feature that we examined. Analysis of the SEM data and morphological comparison to previous isolates shows that OR-10 is identical to previously described Gloeosporium species $[3,13]$. This work illustrates that disparities can and sometimes do exist between morphological observations on an organism and ITS and $18 \mathrm{~S}$ sequence information. Therefore, it is critical that as many observations as possible be made on an organism before claims of identity are determined, especially as related to ecological and physiological processes.

\section{Production of VOCs and bioactivity of Gloeosporium sp.}

Sampled head space GC/MS of Gloeosporium sp. revealed the presence of terpene and furan derivatives, many of which have been observed as potential fungal inhibitors (Table 1). The most relatively abundant of these compounds was 6-Pentyl-2H-pyran-2-one, which constituted $68.5 \%$ of the total VOCs produced by the endophyte (Table 1 ). This compound and its analogs have been observed in Trichoderma species and have been shown as useful fungal inhibitors [8, 11]. When Gloeosporium sp. was grown for 10 days at $23^{\circ} \mathrm{C}$ on PDA, the VOCs of the endophyte proved to be inhibitory to several fungi, including Phytophthora palmivora, Rhizoctonia solani, and Ceratocystis ulmi, which had the least growth of the pathogens tested over a 48 hour test period (Table 2). Furthermore, high inhibition was observed in Colletotrichum lagenarium, Verticillium dahlia, Botrytis cinerea, Sclerotinia sclerotiorum, and Pythium ultimum (Table 2). The test organisms Trichoderma viridae, Cercospora beticola, Geotrichium candidum, Aspergillus fumigatus, and Fusarium solani were only slightly inhibited when tested against 
Gloeosporium sp. (Table 2). The compound 2-pentyl-furan, which accounts for $10.8 \%$ of the VOCs produced by Gloeosporium sp. (Table 1), is also produced by Aspergillius sp. and Fusarium sp., which may explain the resilience exhibited by these species in the presence of the VOCs produced by Gloeosporium sp. [20]. Trichoderma sp. also produces VOCs similar to those emitted by Gloeosporium sp., which also may relate to the limited inhibition seen in the Aspergillius fumigatus and Fusarium solani.

\section{The function of exogenous organics as synergistans and the $\mathrm{mSE}_{50}$}

Certain exogenously applied VOCs displayed a synergistic inhibitory effect against certain fungal plant pathogens when coupled with the VOCs of Gloeosporium sp. (Fig. 2 \& 3). The organisms giving the best response were Ceratocystis ulmi, Rhizoctonia solani and Botrytis cinerea. Initially, allyl acetate, $\mathrm{n}$-decyl acetate, hexyl acetate, isobutyl acetate, isoamyl acetate, and phenethyl acetate were tested to see if increased inhibition was possible against the test fungi by these exogenously applied esters. Of these six esters, only allyl acetate, n-decyl acetate, isoamyl acetate, and phenethyl acetate attained the characteristics of synergism when tested with these pathogens. To ensure that the effect was synergistic, a corresponding amount of ester was assayed alone against the test organisms in the absence of Gloeosporium sp. to determine if it possessed inhibitory activity (Fig. 2B). The bioassay using isoamyl acetate in the absence of Gloeosporium sp. was then compared to the bioassay using both the ester and Gloeosporium sp. by a two-tail T-test, which showed significance at $\mathrm{P}<0.05$ for concentrations ranging from 0.5 to $4 \mu \mathrm{l}$ (Fig. 2A). In the lowest amounts tested (i.e. 0.5-4.0 $\mu \mathrm{l}$ ), these esters had either no effect on the test organism or some growth enhancement when examined in the 
absence of Gloeosporium sp., indicating that the test ester by itself is not inhibitory to the test fungus in these amounts (Fig. 2B). Generally, it was only after a higher amount of the ester (alone) was applied in the bioassay that any inhibition was noted in the test organisms (Fig. 2B). However, when combined with the Gloeosporium sp., the inhibitory synergistic effects were noted at relatively low amounts of the ester (Fig. 2A).

Based on the observations noted above, it was possible to develop a mathematical expression of the "median Synergistic Effect" of a compound by the following description: "The median Synergistic Effect $\left(\mathrm{mSE}_{50}\right)$ is represented by the amount of synergistan needed to bring about an added $50 \%$ increase in inhibition of any test organism over that observed with either of the synergistans tested alone under prescribed test conditions." The value is obtained by plotting growth of the test organism vs. the amount of synergistan in the presence of the inhibitory fungus, i.e. Gloeosporium sp. The points on each plot represent the average values obtained in at least three separate determinations for the inhibition by the putative synergistan. An evaluation was made of the amount of synergistan required to further reduce the growth of the test organism by $50 \%$, thus exhibiting a $50 \%$ increase in growth inhibition above and beyond that produced by the inhibitory fungus alone. This value is best obtained when there is minimal to no effect of the synergistan itself on inhibition of the test organism. Otherwise, any inhibitory activity of the synergistan itself will need to be taken into account. As an example, the test organism, Ceratocystis ulmi, gave no reaction to any of esters when up to $8 \mu \mathrm{l}$ were used alone, but in the presence of Gloeosporium sp., there was an observable synergistic effect at low amounts ranging from 0.5-4 $\mu$. As a result, it was not necessary to subtract any inhibitory effect of any of the ester by itself on the growth of $C$. ulmi in order to 
calculate the $\mathrm{mSE}_{50}$. Ultimately, the $\mathrm{mSE}_{50}$ values for the tested compounds showed that isoamyl acetate produced the best synergistic effect on the test organisms by virtue of possessing the lowest $\mathrm{mSE}_{50}$ values, followed by ally acetate and isobutyric acid on the test organisms (Table 3). Overall, the $\mathrm{mSE}_{50}$ does represent a workable mathematical expression of the potential comparative synergistic value for a test compound. Thus, it can be concluded, the lower the $\mathrm{mSE}_{50}$, the better the synergistic effect.

A previously described bioactive endophytic fungus Muscodor albus produces isoamyl acetate, which has been shown to contribute to the activity of the fungus $[7,17,19]$. Thus, by supplementing the bioassay with isoamyl acetate, a higher inhibition level was achievable, due to the synergism seen between the ester and the VOCs of Gloeosporium sp. (Fig. 2A). This effect is apparent when comparing the amount of inhibition observed in the presence and absence of the ester (Fig. 2, Table 3).

Data from GC/MS shows that Gloeosporium sp. does not produce any carboxylic acids, a component that has been shown to increase the bioactivity of endophytic fungi (Table 1) [7, 19]. Isobutyric acid, a carboxylic acid found in the VOCs produced by M. albus, was added in increasing amounts to bioassay tests, and it, too, increased the inhibition of certain pathogens in a synergistic manner (Fig. 3A, Table 3).

Finally, to this end, isoamyl acetate and 6-pentyl-2H-pyran-2-one were tested in combination in the bioassay, resulting in greater inhibitory activity in combination than that observed when the compounds were tested alone, confirming the observations in Figure 2 (Schiable and Strobel, unpublished observations). The same effect was also noted when 
isobutyric acid was used in the bioassays (Schaible and Strobel, unpublished observations). Thus, these compounds would all be considered synergistans.

\section{Discussion}

The endophyte OR-10 was isolated from small stems of Tsuga heterophylla (Fig. 1). The ITS5.8 and $18 \mathrm{~S}$ phylogenetic data described the organism as most similar to Hypocrea and Trichoderma species, but cultural characteristics along with scanning electron micrographs of the fruiting organism showed conflicting data to the $18 \mathrm{~S}$ and ITS sequence information. The morphology of OR-10 grown on PDA for 10 days varies widely from that of Trichoderma viridae grown on PDA for 10 days. OR-10 grows close to the surface of the PDA agar and maintains a white appearance, while Trichoderma viridae sends hyphae upwards and, after 5 days, begins to turn green due to spore formation. Though the organisms are similar based on the nucleotide level of two alleles and secondary volatile products formed, they appear to be distinctly different based on morphology (Table 1). Because of the distinct differences in morphology between OR-10 and the fungal species Hypocrea and Trichoderma, the fungal endophyte was characterized as a member of the acervuli-forming Gloeosporium group. This finding, in conjunction with previous research, has shown that fungal species can be misclassified by their ITS and 18 S genes [6, 28]. Limiting the phylogenetic analysis to one or two genes neglects the majority of the genome, providing poor resolution of genetic diversity within the organism. This needs to be taken into account especially by those workers who are doing metagenomic studies and then proceeding to make broad statements about the identity of varioius microorganisms without ever examining them. 
Strong organic odors produced by this fungus are primarily attributed to a mixture of 2-pentylfuran and 6-pentyl-2H-pyran-2-one (Table 1). Biological activity tests using Gloeosporium sp. showed impressive inhibition against an array of pathogenic fungi, including Phytophthora palmivora, Botrytis cinerea, Rhizoctonia solani and Ceratocystis ulmi. It has been previously demonstrated that esters and small organic acids are often a major contributor to the biological activity of VOCs produced by fungi, as seen in Muscodor albus [17]. Since Gloeosporium sp. did not produce any detectable esters or acids, it was hypothesized that the addition of individual esters or acids would result in increased biological activities in the inhibition tests as per earlier studies [19]. Subsequently, testing of the endophyte and the additional individual esters allyl acetate, n-decyl acetate, isoamyl acetate, and phenethyl acetate against Botrytis cinerea, Rhizoctonia solani and Ceratocystis ulmi showed increased inhibition (Fig. 2). While the addition of either hexyl acetate or isobutyl acetate resulted in no increased inhibition, each of the other esters that were tested showed enhanced biological activity or a synergistic effect. Virtually the same enhanced inhibitory effect was noted upon the addition of isobutyric acid to the bioassay test (greater than with isobutyric acid alone) (Fig. 3). It became obvious that some exogenously added organic compounds were capable of producing added inhibitory (synergistic) effects with the VOCs of Gloeosporium sp. against the test organisms (Figs. 2 \& 3).

In a comparable case, Oidium sp. produces a wide-ranging mixture of esters, and enhanced biological activity (synergism) of the VOCs of this organism was noted with the addition of isobutyric acid [19]. It is obvious that some esters, small organic acids and other volatiles have the potential to act synergistically, resulting in greatly enhanced inhibitory or antimicrobial 
activities. In a manner that provides new meaning to substances that have this chemical/biological potential, we a novel and descriptive name is proposed to such compounds: "synergistans". Some compounds that have been tested that fit this description, besides the examples in this report, are propanoic acid with isoamyl hexanoates (Strobel, unpublished), isobutyric acid with isoamyl acetate (Strobel, unpublished), and a mixture of esters with isobutyric acid [19].

Synergistans are widely present in nature. For example, isobutyric acid and isoamyl acetate are volatile products of $M$. albus and a number of other isolates of this fungus $[7,10,17]$. Furthermore, in cases with other species of Muscodor, such as M. crispans and M. sutura, isobutyric acid is present and so, too, are a plethora of esters that undoubtedly enhance the biological activity of these fungal species by virtue of one or more synergistic activities [10]. From a physiological and ecological perspective, it is easy to imagine that plants inhabited with endophytes making compounds behaving as synergistans may be provided protection from pathogenic microbes that are sensitive to these compounds (Table 2). Synergistans hold promise in helping to control unwanted microorganisms from agricultural, industrial and medical situations, as well as for replacing overly-used antibiotics in these settings. 


\section{References}

1. Ahamed A, Ahring BK (2011). Production of hydrocarbon compounds by endophytic fungi Gliocaldium sp. grown on cellulose. Bioresource Tech 102: 9718-9722

2. Altschul SF, Madden TL, Schaffer AA, Zhang J, Zhang Z, Miller W, Lipman DJ (1997). Gapped BLAST and PSI-BLAST: a new generation of protein database search programs. Nucleic Acids Res 25: 3389-3402

3. Anselmi, N. (1980). Studies on Drepanopeziza salicis (Tull.) v. Höhnel, perfect state of Gloeosporium salicis West. European journal of forest pathology, 10(7), 438-447.

4. Banerjee, D. Strobel, G.A. Booth, E., Geary, B., Sears, J., Spakowicz, D., and Busse, S. (2010). An endophytic Myrothecium inundatum producing volatile organic compounds. Mycosphere 3: 241-247

5. Castillo UF, Browne L, Strobel GA, Hess WM, Ezra S, Pacheco G, and Ezra D (2007). Biologically active endophytic Streptomycetes from Nothofagus spp. And other plants in Patagonia. Microbial Ecology 53.1: 12-19

6. Cruz, D., Suárez, J. P., Kottke, I., Piepenbring, M., \& Oberwinkler, F. (2011). Defining species in Tulasnella by correlating morphology and nrDNA ITS-5.8 S sequence data of basidiomata from a tropical Andean forest. Mycological Progress, 10(2), 229-238.

7. Ezra D, Hess WM \& Strobel GA (2004). New endophytic isolates of Muscodor albus, a volatile antibiotic producing fungus. Microbiology 150: 4023-4031

8. Hanson JR (2005). The Chemistry of the Bio-Control Agent, Trichoderma harzianum. Science Progress, 88(4): 237-248.

9. Kudalkar P, Strobel GA, Riyaz-UI-Hassan S, Geary B, Sears J (2011). Muscodor sutura, a novel endophytic fungus with volatile antibiotic activities. Mycoscience 53:319-325

10. Mitchell, A.M. Strobel, G.A., Moore, E., Robison, R., and Sears, J. (2010). Volatile antimicrobials from Muscodor crispans. Microbiology 156: 270-277.

11. Parker SR, Cutler HG, Jacyno JM, and Hill RA (1997). Biological activity of 6-Pentyl-2Hpyran-2-one and its analogs. Journal of Agricultural Food Chemistry 45: 2774-2776

12. Rodrigues FFG, Costa JGM, Coutinho HDM (2009). Synergy effects of the antibiotics gentamicin and the essential oil of Croton zehnteri. Phytomedicine 16: 1052-1055 
13. Sharples, R. O. (1959). Observations on the perfect state of $<i>$ Gloeosporium perennans $</ i>$ in England. Transactions of the British Mycological Society, 42(4), 507IN8.

14. Strobel GA, (2006). Harnessing endophytes for industrial microbiology. Current Opinion in Microbiology 9.3: 240-244

15. Strobel GA, Daisy B (2003). Bioprospecting for Microbial Endophytes and Their Natural Products. Microbiology and Molecular Biology Reviews 67.4: 491-502.

16. Strobel GA, Daisy B, Castillo U \& Harper J (2003). Natural Products from Endophytic Microorganisms. American Chemical Society and American Society of Pharmacognosy 10: 1021

17. Strobel GA, Dirksie E, Sears J \& Markworth C (2001). Volatile antimicrobials from Muscodor albus, a novel endophytic fungus. Mirobiology 147: 2943-2950

18. Strobel GA, Knighton B, Kluck K, Ren Y, Livinghouse T, Griffin M, Spakowicz D \& Sears J (2008). The Production of Myco-diesel Hydrocarbons and Their Derivatives by the Endophytic Fungus Gliocladium Roseum (NRRL 50072). Microbiology 154.11: 3319-328.

19. Strobel, G. A., Spang, S., Kluck, K., Hess, W. M., Sears, J., \& Livinghouse, T. (2008). Synergism among volatile organic compounds resulting in increased antibiosis in Oidium sp. FEMS microbiology letters, 283(2), 140-145.

20. Syhre M, Scotter JM \& Chambers ST (2008). Investigation into the production of 2Pentylfuran by Aspergillus fumigatus and other respiratory pathogens in vitro and human breath samples. Medical Mycology 46: 209-215

21. Tomsheck A, Strobel GA, Booth E, Geary B, Spakowicz D, Knighton B, Floerchinger C, Sears J, (2010). Hypoxylon sp an endophyte of Persea indica producing 1, 8 -cineole and other bioactive volatile with fuel potential. Microbial Ecology 60: 903-914

22. Veras HNH, Rodrigues FFG, Colares AV, Menezes IRA, Coutinho HDM, Botelho MA, Costa JGM (2012). Synergistic antibiotic activity of volatile compounds from the essential oil of Lippia sidoides and thymol. Fitoterapia 83: 508-512

23. Vermaa VC, Kharwara RN \& Strobel GA (2009). Chemical and Functional Diversity of Natural Products from Plant Associated Endophytic Fungi. Natural Product Communications 4: 1-22.

24. Wagner H, Ulrich-Merzenich $\mathrm{G}$ (2009). Synergy research: Approaching a new generation of phytopharmaceuticals. Phytomedicine 16: 97-110 
25. White, T. J., Bruns, T., Lee, S. J. W. T., \& Taylor, J. W. (1990). Amplification and direct sequencing of fungal ribosomal RNA genes for phylogenetics. PCR protocols: a guide to methods and applications, 18, 315-322.

26. White TJ, Bruns T, Taylor JW (1990). Amplification of direct sequencing of fungal ribosomal RNA genes for phylogenetics. In: Innis MA, Gelfand DH, Sninsky JJ, White TJ (eds) PCR protocols: a guide to methods and applications. Academic Press, San Diego, pp 315-324

27. Wilkinson HH, Siegel MR, Blankenship JD, Mallory AC, Bush LP \& Schardl CL (2000). Contribution of Fungal Loline Alkaloids to Protection from Aphids in a Grass-Endophyte Mutualism. Molecular-Plant Microbe Interactions 13.10: 1027-1033

28. Xie, J., Strobel, G. A., Mends, M. T., Hilmer, J., Nigg, J., \& Geary, B. (2013). Collophora aceris, a Novel Antimycotic Producing Endophyte Associated with Douglas Maple. Microbial ecology, 66(4), 784-795. 
Figure 1. Scanning electron micrographs showing the fruiting structures and spores of Gloeosporium sp. (OR-10) grown on water agar with its host plant, Tsuga heterophylla. A and B. The acervulus of Gloeosporium sp. $(450 \times 500 \mu \mathrm{m})$ forming on plant host materials. C and D. The spore size measured $3 \times 4 \mu \mathrm{m}$ forming on condiophores. It appears that the spore wall is fragile, and the micrograph fixation process caused the collapse of the individual spores, resulting in their bowl shape. Spores circled in D are examples of spores that were more or less capable of withstanding the fixation process and did not totally collapse.
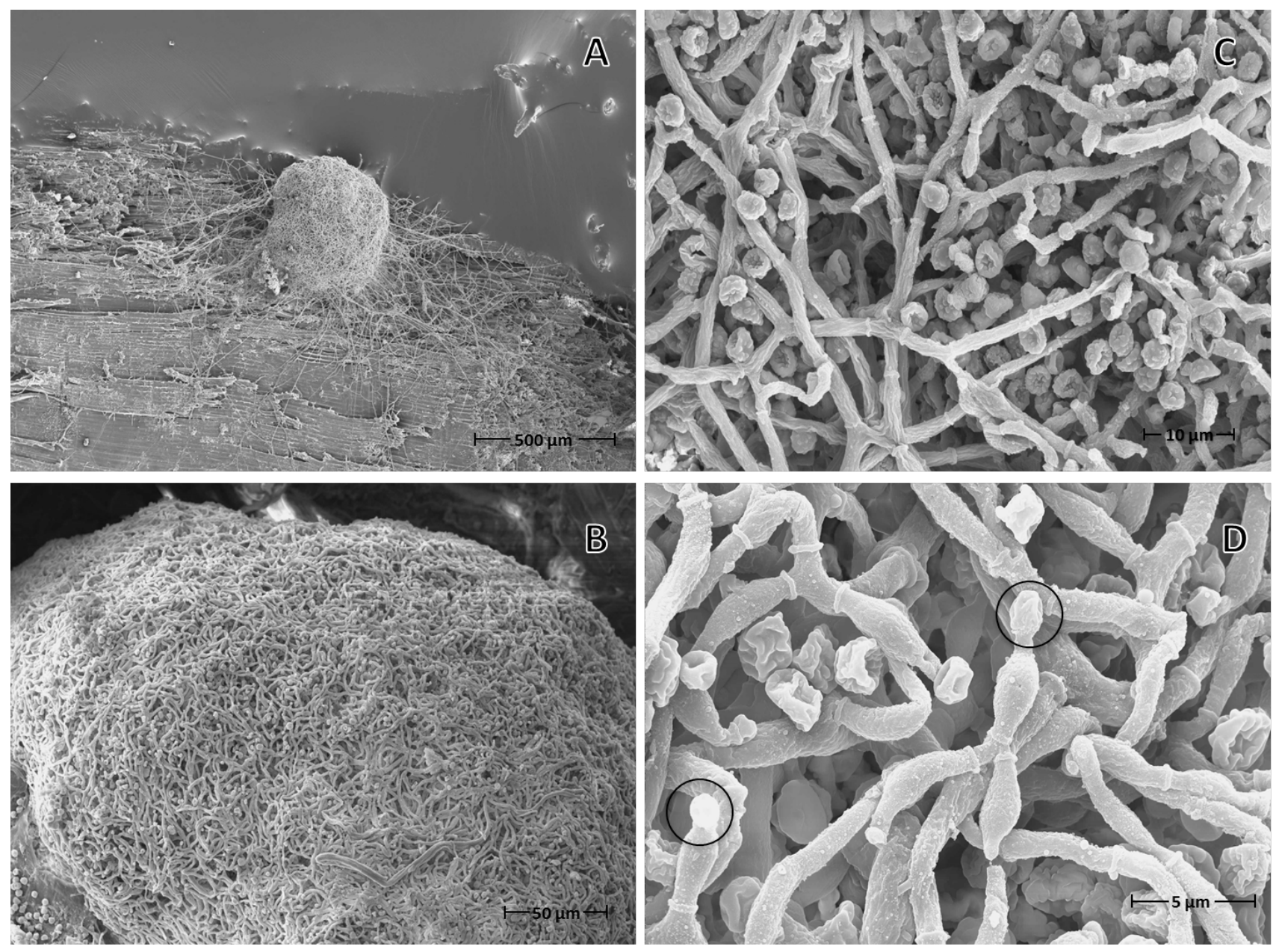
Figure 2. The synergistic effect seen between isoamyl acetate and Gloeosporium sp. Half-moon plate bioassays were conducted with Gloeosporium sp., according to the methods described. The test organisms were Rhizoctonia solani, Botrytis cinerea, and Ceratocystis ulmi. In the gap between the two half-moons of PDA, a sterile microwell was placed, into which a corresponding volume of isoamyl acetate was added. This assay was conducted for a full 48 hours, after which the growth of the pathogen hyphae was measured. (A) Illustrates the synergistic effect seen when isoamyl acetate was added to the microwell in increasing amounts on the plates containing the ten-day old OR-10. (B) Isoamyl acetate was added to the microwell in the absence of OR-10. The control represents the growth of the pathogens on PDA in the absence of both isoamyl acetate and OR-10. The pathogens were also tested against the Gloeosporium sp. endophyte OR-10 (columns denoted by OR-10) in the absence of the isoamyl acetate for comparison. To determine if the synergistic effect between the synergistan and Gloeosporium sp. was statistically different, a two tail T-test was used to compare the results in graph $\mathbf{A}$ to those in graph $\mathbf{B}$. Tests where there was a relevant statistical difference $(P<0.05)$ between the inhibitory effect against the test organism by the ester alone (graph B), and by the synergistic effect of the synergistan and Gloeosporium sp. (graph A), are indicated by an asterisk $\left({ }^{*}\right)$. Tests where the ester alone was capable of completely inhibiting the growth of the test organism are denoted by a dagger $(\dagger)$, as shown in graph $\mathbf{B}$. In these cases, the tests are not considered statistically different and therefore in graph $\mathbf{A}$ these tests lack asterisks.

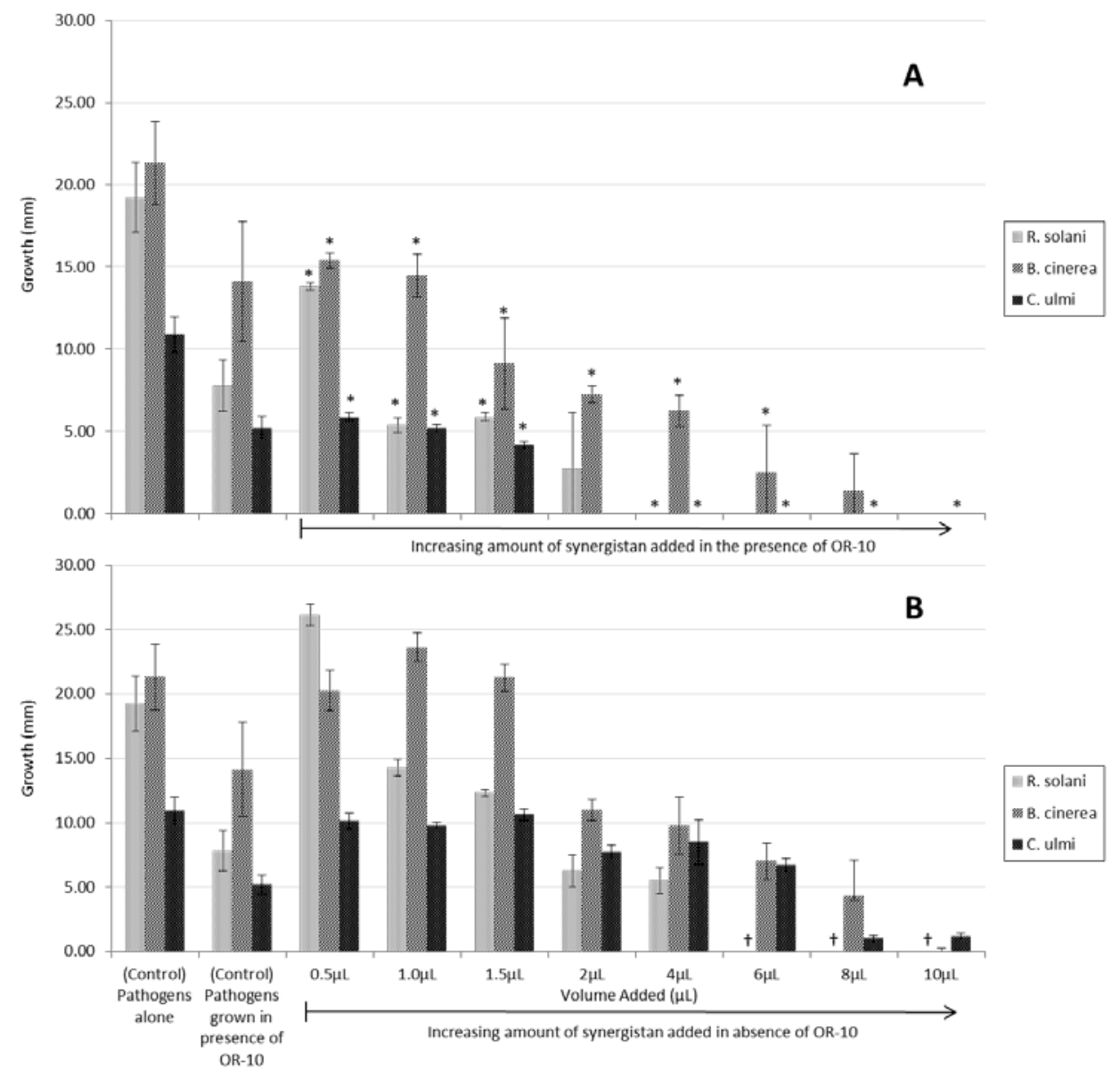


Figure 3. The synergistic effect seen between isobutyric acid and the endophyte OR-10. The same method used for the isoamyl acetate test was employed to test the synergistic effects of isobutyric acid. A. The synergistic effect of isobutyric acid and the endophyte OR-10. B. The results showing pathogens grown exclusively in the presence of isobutyric acid. The Control was the growth of the pathogens when grown on PDA in absence of both isoamyl acetate and OR10. The pathogens were also tested against the Gloeosporium sp. endophyte OR-10 (columns denoted by OR-10) in the absence of the isoamyl acetate for comparison. As described in Figure 2 , asterisks $\left({ }^{*}\right)$ refer to $p$-values and dagger $(+)$ refer to full inhibition of test organism by ester alone.

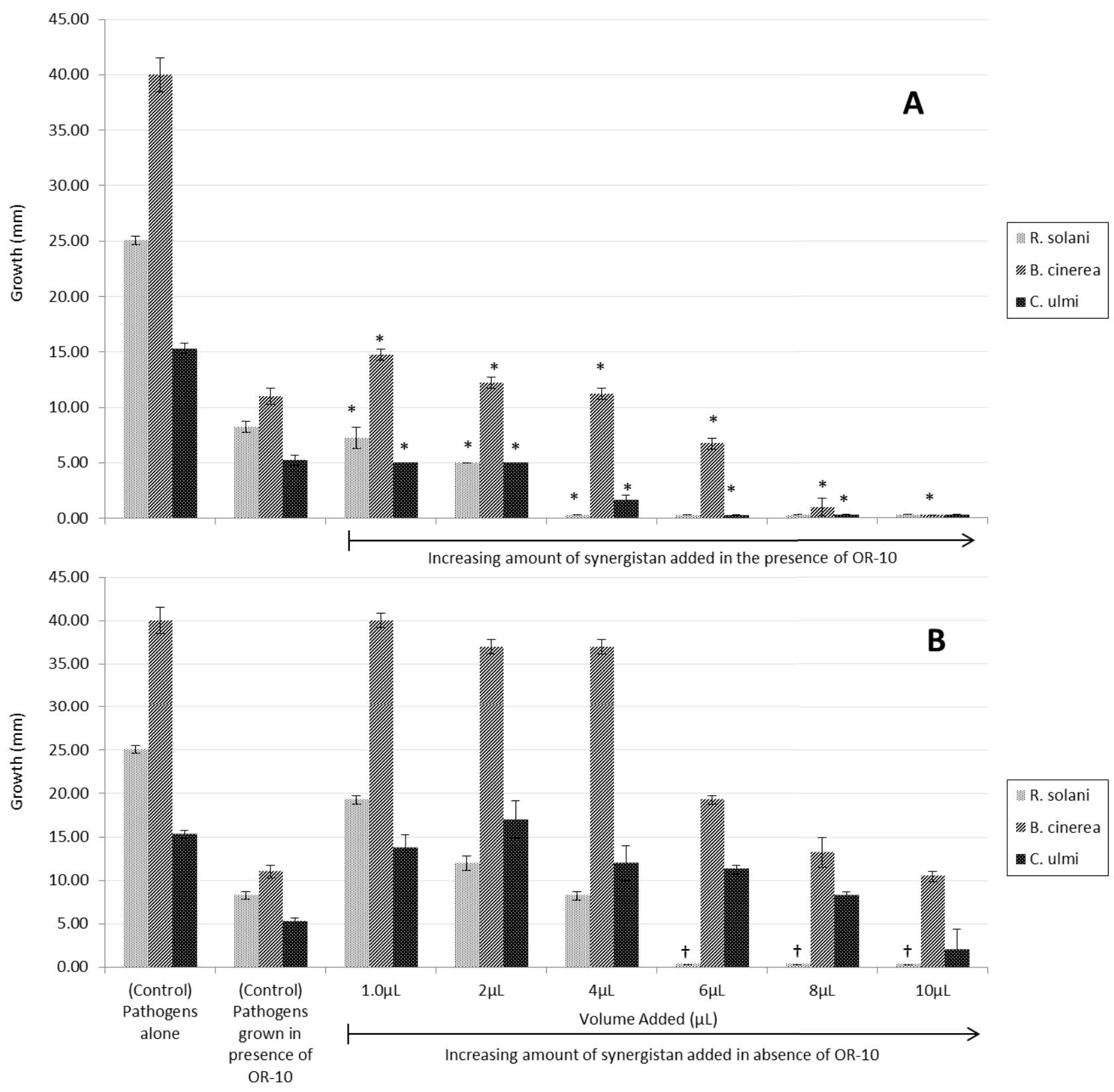


Table 1. A GC/MS head space analysis of the volatile compounds produced by Gloeosporium sp. after 10 days of incubation at $23^{\circ} \mathrm{C}$ on PDA. Compounds that were also present in a control PDA plate have been removed from the analysis so only the VOCs produced by OR-10 are shown. Compounds with a quality match of less than $70 \%$ are listed as unknown. Abbreviations used: RT (retention time in minutes), RA (relative area), MW (molecular weight).

\begin{tabular}{cclccc}
\hline RT & RA & \multicolumn{1}{c}{ Possible Compound } & MW & Quality & \% Volume \\
\hline 11.54 & 17.475 & Furan, 2-pentyl- & 138 & 94 & 12.66 \\
17.04 & 0.384 & 2-n-Heptylfuran & 166 & 95 & 0.28 \\
20.82 & 1.016 & 2-Norpinene & 204 & 91 & 0.74 \\
30.78 & 0.286 & Nerolidol 2 & 222 & 91 & 0.21 \\
33.79 & 94.532 & 2H-Pyran-2-one, 6-pentyl- & 166 & 91 & 68.46 \\
& 24.391 & Unknown & & $<70$ & 17.66
\end{tabular}


Table 2. Effects of a 12-day old culture of Gloeosporium sp. VOCs on fungal pathogens. Inhibition values were calculated as a percentage of growth inhibition when compared to an untreated control test organism after 48 hours of exposure. Tests were conducted in triplicate and results varied as indicated by standard deviations. The pathogens were still viable once removed from the test plate after the two day incubation period and were not killed by the VOCs of Gloeosporium sp. No organisms were completely killed by the VOCs from OR-10. Species with an asterisk $(*)$ next to them were used in the synergy tests.

\begin{tabular}{|c|c|c|}
\hline Test Microbe & $\begin{array}{c}\% \text { Inhibition against control } \\
\text { after } 2 \text { days }\end{array}$ & Pathogen fatalities \\
\hline Aspergillus fumigatus & $15.2 \%( \pm 7.2)$ & \\
\hline Botrytis cinerea* & $54.4 \%( \pm 35.8)$ & \\
\hline Ceratocystis ulmi* & $75.5 \%( \pm 36.2)$ & \\
\hline Cercospora beticola & $15.0 \%( \pm 11.6)$ & \\
\hline Colletotrichum lagenarium & $48.0 \%( \pm 41.1)$ & \\
\hline Fusarium solani & $6.9 \%( \pm 5.3)$ & \\
\hline Geotrichium candidum & $22.0 \%( \pm 11.8)$ & \\
\hline Phytophthora palmivora & $100.0 \%$ & Not killed \\
\hline Phytopthera cinnamomi & $17.9 \%( \pm 15.3)$ & \\
\hline Pythium ultimum & $33.1 \%( \pm 28.0)$ & \\
\hline Rhizoctonia solani* & $83.3 \%( \pm 33.4)$ & \\
\hline Sclerotinia sclerotiorum & $37.3 \%( \pm 7.9)$ & \\
\hline Trichoderma viridae & $9.4 \%( \pm 5.1)$ & \\
\hline Verticillium dahliae & $48.2 \%( \pm 43.2)$ & \\
\hline
\end{tabular}


Table 3. The $\mathrm{mSE}_{50}$ values calculated for the synergisitic effect of various synergistans on the growth of a series of test organisms. The $\mathrm{mSE}_{50}$ is the amount ( $\left.\mu \mathrm{l}\right)$ of synergistan needed to bring about an additional $50 \%$ inhibition of the growth of the test organism under the conditions outlined in the Materials \& Methods. The values presented are the best estimates made by plotting growth vs. amount of the test compound placed in the assay. The test using the ester decyl acetate (alone) against Rhizoctonia solani resulted in the test organism exhibiting a greater inhibition than the synergistic effect of the ester and Gloeosporium sp, thus no $\mathrm{mSE}_{50}$ could be calculated.

$\mathrm{mSE}_{50}$ in $\boldsymbol{\mu l}$ for the Test Organism

$\begin{array}{lccc}\begin{array}{l}\text { Compound tested as } \\ \text { a synergistan }\end{array} & \begin{array}{r}\text { Rhizoctonia solani } \\ \text { Al }\end{array} & \text { Botrytis cinerea } & \text { Ceratocystis ulmi } \\ & & \mu \mathrm{l} & \mu \mathrm{l} \\ \text { Allyl acetate } & 1.84 & 6.16 & 1.29 \\ \text { Decyl acetate } & \mathrm{N} / \mathrm{A} & 8.29 & 0.38 \\ \text { Isoamyl acetate } & 2.00 & 2.54 & 1.69 \\ \text { Phenethyl acetate } & 1.73 & 14.03 & 0.40 \\ \text { Isobutyric acid } & 3.17 & 7.40 & 4.72\end{array}$

